\title{
Pierre Auger et Lise Meitner Comparaison de leurs contributions à l'effet Auger
}

Olivier Hardouin Duparc (olivier.hardouinduparc@polytechnique.edu)

Unité mixte de Physique CNRS/CEA/X LSI, École Polytechnique, 91128 Palaiseau

Lise Meitner a observé et décrit l'effet Auger quelques mois sans doute avant

Pierre Auger, en explicitant une suggestion des

Britanniques Ellis

et Rutherford.

Mais sa préoccupation

était tout autre que celle

de Pierre Auger et c'est donc naturellement que l'appellation « effet Auger » est apparue, d'abord en Allemagne.

Olivier Hardouin Duparc rappelle et commente ici quelques détails de cette histoire ${ }^{(1)}$.
Lorsqu'une particule incidente de forte énergie (photon $\mathrm{X}$ ou $\gamma$ ou électron très rapide) éjecte du cortège électronique d'un atome un électron d'une couche profonde, un électron plus externe descendra sur ce niveau ; la différence d'énergie pourra soit être émise en tant que rayon $\mathrm{X}$, c'est de la fluorescence $\mathrm{X}$, soit provoquer l'éjection d'un autre électron orbital que l'on appelle électron Auger (fig. 1) [1-3]. Le rapport de fréquences entre ces deux possibilités dépend du numéro atomique de l'atome.
Pour les atomes plus légers que le gadolinium, il est en faveur du processus Auger. L'énergie du deuxième électron est fonction de la seule nature de l'atome (l'énergie de la particule incidente n'intervient plus), et sa mesure peut donc servir de moyen d'investigation spectroscopique.

La spectroscopie Auger est utilisée aussi bien à des fins de recherche fondamentale que de caractérisation dans l'industrie.

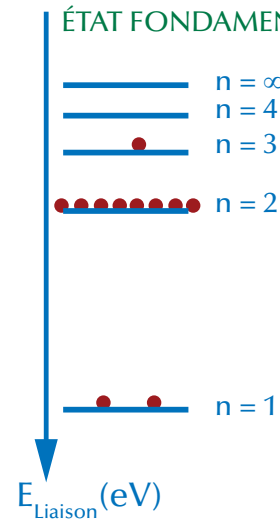

A

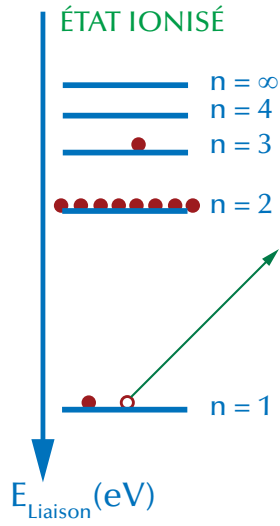

B

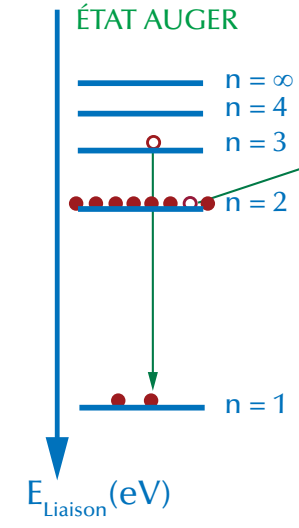

C

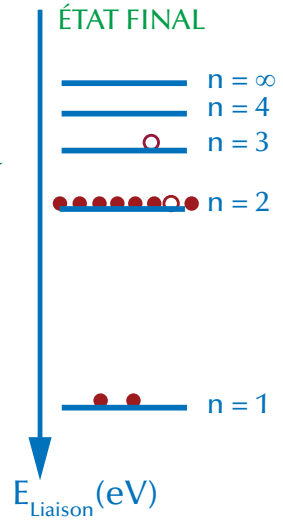

$\mathrm{D}$

1. Schéma simplifié de l'effet Auger dans un atome de sodium (11 électrons). En première approximation, l'énergie cinétique de l'électron Auger $\mathrm{E}_{(}(\mathrm{e})$ s'obtient en faisant le bilan entre l'état $C$ et l'état $B$ : remplissage de la lacune en $n=1$ par un électron moins énergétique $n=3$; transfert du gain d'énergie de liaison $E(n=1)-E(n=3)$ à un électron du niveau $n=2$, qui est éjecté avec l'énergie totale $E(n=2)+E_{c}\left(e^{-}\right)$. D'où $E_{(}\left(e^{-}\right)=E(n=1)-[E(n=2)+E(n=3)]$. Les spécialistes noteront $K, L, M$, N les niveaux $n=1,2,3$, 4 et subdiviseront L en $L_{1}(2 s)$ et $L_{23}(2 p)$ (voire $L_{2}\left(2 p^{1 / 2}\right)$ et $L_{3}\left(2 p^{3 / 2}\right)$. Ils distingueront aussi entre les niveaux de l'atome ionisé et les niveaux de l'atome neutre. Pour un atome situé à la surface d'un solide métallique, il faut rajouter le travail de sortie du métal (traditionnellement noté -e $\Phi)$. Si l'atome est à une profondeur supérieure au libre parcours moyen de l'électron, il faut rajouter des pertes d'énergie par collisions. (Remarque : Auger a d'abord utilisé l'argon qui comporte 18 électrons, car c'est un gaz rare peu réactif).

- : État électronique occupé. O : Lacune électronique.

(Source : www.physique.unicaen.fr/ tice/new_site_tice/pdf/cours_Int_ray_mat/apprendre/B/III/2/atome_III_22.html) 


\section{Lise Meitner et laffaire des rayons bêta nucléaires}

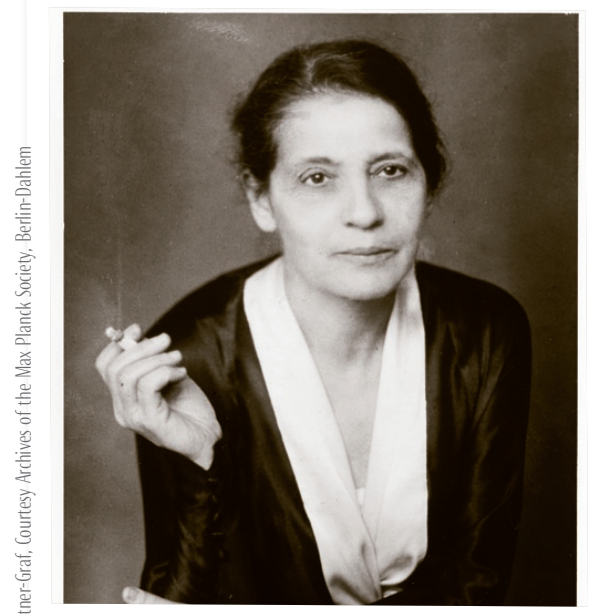

Il existe de nombreuses biographies de Lise Meitner. Née en 1878 en Autriche, elle alla à Berlin en 1907 pour étudier avec Max Planck et commença à travailler sur la radioactivité des émetteurs de rayons $\beta$ avec Otto Hahn dans un barraquement en bois appartenant à l'institut de chimie de l'université de Berlin. Bien entendu, d'autres équipes effectuaient le même type de recherches et, l'affaire n'étant pas simple, ainsi qu'on va le voir, des divergences d'interprétation surgirent assez vite. Cette histoire, complexe et fascinante, a été racontée plusieurs fois [4-7], et avec un maximum de détails par Carl Jensen [7].

Mus par l'idée apparemment très raisonnable d'une simple analogie entre les rayons $\beta$ et les rayons $\alpha$, Lise Meitner et Otto Hahn avaient d'abord pensé avoir démontré, à l'aide d'un spectromètre magnétique et de plaques photographiques, que les $\beta$ sont purement monoénergétiques (donnent un spectre de pics discrets). En 1913 cependant, James Chadwick montrait, en utilisant un compteur Geiger plutôt que des plaques photographiques, que les $\beta$ exhibent en outre une distribution continue. Chadwick travaillait également à Berlin, avec Hans Geiger auprès duquel Ernest Rutherford l'avait recommandé puisque Geiger avait d'abord été son assistant à Manchester (les relations entre l'Angleterre et l'Allemagne étaient très bonnes avant la Première Guerre mondiale [8]).
Les recherches continuèrent après la Guerre, avec Lise Meitner à Berlin et avec le groupe de Rutherford à Manchester, et une controverse assez vive eut lieu en 1920-22. Charles Drumond Ellis, un étudiant de Rutherford (Sir Ernest Rutherford depuis 1914), pensait et écrivait que les pics discrets du spectre des $\beta$ pouvaient probablement être expliqués par l'action de rayons $\gamma$ (d'origine nucléaire) sur les électrons de l'atome (via une sorte d'effet photoélectrique interne) tandis que la partie continue du spectre était certainement d'origine nucléaire, avec un caractère continu probablement dès la sortie du noyau, même s'il n'avait aucune idée de comment expliquer cela. Tout au contraire, Lise Meitner essayait de sauvegarder coûte que coûte son hypothèse de monochromaticité des rayons $\beta$ nucléaires. Tandis qu'elle arrivait à préciser l'explication des pics discrets en termes de processus atomiques internes, incluant l'effet qu'elle appellera elle-même plus tard l'effet Auger, elle émit l'idée que le spectre continu était dû à des $\beta$ nucléaires monochromatiques perturbés par diverses causes... On a donc, avec Ellis (et Rutherford) en 1921 et 1922, et Meitner en 1922 et 1923, une première description de processus de conversion d'énergie entre couches atomiques avec émission d'électrons appelés $\beta$ secondaires.

Mais l'intérêt de ces auteurs était clairement ailleurs : il concernait la nature des $\beta$ primaires, d'origine nucléaire et sur ce point leurs opinions divergeaient ; Meitner tînt même des propos assez polémiques en 1923. Malheureusement pour elle, il se révéla finalement que ses concurrents avaient raison, même s'ils ne pouvaient expliquer pourquoi.

La simple amélioration progressive de la précision des spectromètres magnétiques et des compteurs Geiger ne put suffire à convaincre Lise Meitner, et Ellis conçut un nouveau type d'expérience, de type calorimétrique pour mesurer toute l'énergie émise par le noyau (en principe). Les problèmes de précision de détection calorimétrique étaient bien sûr cruciaux et il fallut deux années à Ellis et Wooster pour parvenir à leur fin. Leur résultat corroborait l'assertion d'Ellis selon laquelle les électrons d'origine nucléaire sont émis avec un spectre continu cohérent avec le spectre détecté en Geiger. Meitner essaya d'arguer d'un possible biais dû̀ à de l'énergie emportée par des $\gamma$ et non détectée par le calorimètre. Elle répéta elle-même l'expérience de Ellis et Wooster à l'aide d'un calorimètre amélioré et ne put que confirmer leur résultat. Cette fois elle abandonna la partie, tout en déclarant qu'elle ne comprenait absolument pas la nature de ces résultats. Personne ne les comprenait davantage, pas même Albert Einstein.

Il fallut encore plusieurs années, et des hypothèses parfois désespérées, pour que la vraie solution de l'énigme apparaisse peu à peu : la réaction bêta nucléaire est «neutron $\rightarrow$ proton + électron + antineutrino ", et les antineutrinos emportent de l'énergie mais sont très difficiles à détecter (ils ne le furent qu'en 1956). Notons que Pauli, l'audacieux père, en décembre 1930, de l'hypothèse de la particule neutre indétectable [9] avait, l'année précédente, écrit dans une lettre à Ehrenfest que l'expérience de Ellis et Wooster ne mettait en rien en péril l'interprétation de Lise Meitner. .

Donc, même si elle est tout à fait pardonnable, Lise Meitner ne sortait pas grande vainqueur de cette histoire et de cette controverse.

\section{Références}

1 - M.-G. Barthés-Labrousse, "The Auger Effect", Microscopy Microanalysis Microstructures 6 (1995) 253.

2 • J. Cazaux, « Spectroscopie Auger », dans les Techniques de l'Ingénieur (2007) P2620.

3 - J.-P. Eberhart, Analyse structurale et chimique des matériaux, Dunod, Paris (1997).

4 R.L. Sime, Lise Meitner: A Life in Physics, University of California Press, Berkeley (1996).

5 B. Fernandez, De l'atome au noyau. Une approche historique de la physique atomique et de la physique nucléaire, Ellipses, Paris (2006).

6 - A. Pais, Inward Bound. Of Matter and Forces in the Physical World, Oxford University Press, Oxford (1988)

7 - C. Jensen, Controversy and Consensus, Nuclear beta decay: 1911-1934, Thèse de doctorat (1990), éditée à titre posthume par F. Aaserud, H. Kragh, E. Ründinger et R. H. Stuewer, Birkhaüser, Basel (2000).

$8 \bullet$ 0.B.M. Hardouin Duparc, « Alfred Wilm et les débuts du Duralumin ", Cahiers de l'histoire de l'aluminium 34 (2005) 63.

9 • J.-Cl. Boudenot, « La vie de Wolfgang Ernest Pauli », Reflets de la Physique 12 (2008) 20.

$10 \bullet$ http://picardp1.mouchez.cnrs.fr/Auger.html 


\section{Pierre Auger et les électrons atomiques}

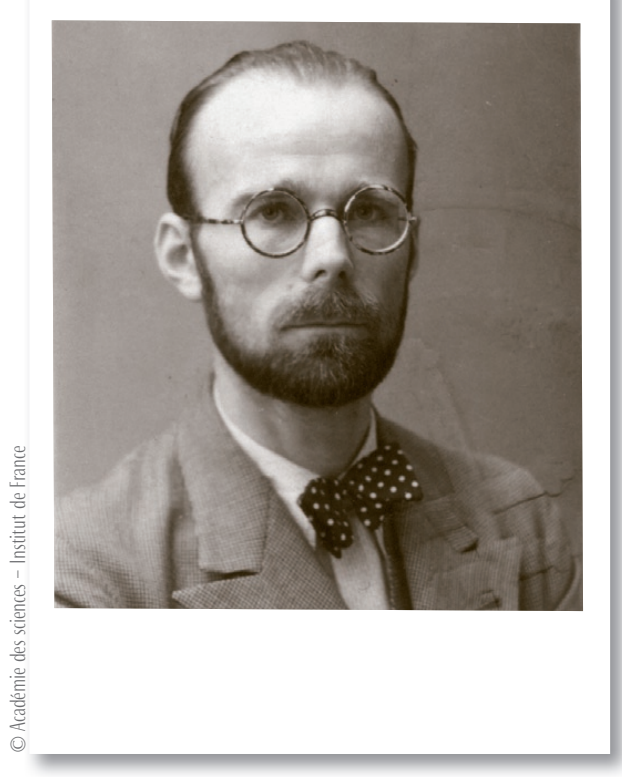

Né en 1899, Pierre Victor Auger intégra l'École normale supérieure en 1919 au sein d'un groupe de biologie, tout en étant également intéressé par la physique atomique [10]. Au moment de faire un stage en laboratoire avant de passer l'agrégation il décida de construire la première chambre à brouillard made in France avec son grand ami à l'École, Francis Perrin. La publication, par C.T.R. Wilson, des premières images quelques années auparavant les avaient fascinés. Ils réussirent à visualiser des traces de particules $\alpha$. Pierre Auger décida de continuer dans cette voie et, ayant obtenu une bourse, il resta dans le laboratoire du père de son ami, le Professeur Jean Perrin, pour y faire sa thèse. Le but était de visualiser tout le processus de la photoexcitation atomique : d'abord la production d'un photoélectron, puis l'émission d'un quantum de rayonnement [en fait un quantum purement virtuel], et l'absorption de ce quantum avec production d'un autre photoélectron, le tout dans la même chambre.

Voici ce qu'écrivait Pierre Auger dans sa première communication à l'Académie des sciences, le 16 juillet 1923 : "Lors $d u$ départ du premier électron, sous forme du rayon $\beta$ secondaire, une place est laissée libre dans le système électronique de l'atome excité. La chute d'un électron plus périphérique sur ce niveau s'accompagne de l'émission d'un quantum de rayonnement caractéristique. Ce quantum peut être absorbé dans l'atome même, et servir à la production, aux dépens des niveaux périphériques, d'un rayon $\beta$ tertiaire: les caractéristiques de ce rayon seront alors celles qui ont été décrites précédemment. La répétition du même processus doit aboutir à la production d'un rayon quaternaire; et je crois en effet avoir observé de tels rayons dans le cas de l'iode."

À ma connaissance c'est en Allemagne (et en allemand) que sont apparues les expressions " électrons Auger " (Auger Elektronen, Gr. Wentzel, alors à l'université de Leipzig, en 1927) et "processus Auger " (Auger Prozesse, Kallmann et Rosen, à Berlin, comme Meitner, en 1931). Lise Meitner elle-même, en 1933, parle de l'effet Auger (ou effet Rosseland-Auger) dans son chapitre de revue sur la structure nucléaire dans le volume XXII de la prestigieuse série Handbuch der Physik éditée par Geiger et Scheel. Dans le titre de son article avec Kronig en 1935, Dirk Coster, un ami proche de Lise Meitner, parle d'un nouveau type d'effet Auger, et, la même année, l'australien E.H.S. Burhop publie un article de treize pages intitulé « l'effet Auger " dans les Proceedings de la Royal Society de Londres.

Pendant ce temps Auger étudiait les rayons cosmiques avec des compteurs de Geiger-Müller et détectait, d'abord rue Pierre Curie avec Roland Maze, les grandes gerbes que l'on appelle désormais gerbes d'Auger. Il devait ensuite se consacrer à la création de divers types d'enseignements (génétique, électronique, les ENSI) ainsi qu'à la fondation d'organismes de recherche tant français (CEA, CNES) qu'européens (CERN, ESRO ${ }^{(2)}$ ). Il écrivit un essai de monadologie, L'Homme microscopique (en 1952, avec un provocateur chapitre sur la biologie du mariage) ainsi qu'un recueil de poèmes courts, et il sculpta également des bronzes.

Pierre Auger décéda en 1993 à l'âge de 94 ans. Lise Meitner s'était éteinte en 1968 à 90 ans. L'élément 109 est appelé le meitnerium.

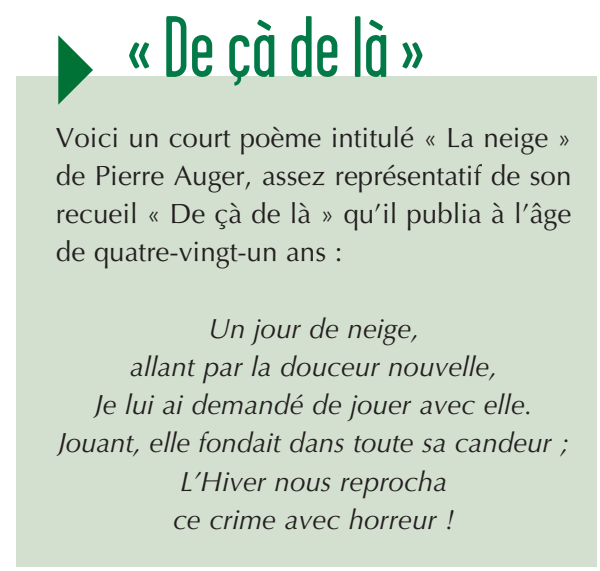

\section{Conclusion}

Lise Meitner, en tant que physicienne du nucléaire, ne se sentait pas très concernée par la physique atomique (ni par la chimie, cf. son attitude vis-à-vis de la médaille Emil Fischer attribuée à Hahn en 1919). En outre, dans ce qui l'intéressait vraiment dans l'affaire des vrais $\beta$ (nucléaires), son interprétation s'était finalement révélée erronée.

À l'inverse, pour Pierre Auger, il s'agissait de son travail de thèse. Il avait largement atteint son but et il avait ainsi rendu " visibles " ces électrons, au moins sous forme de traces, plus " parlantes ", en un certain sens, que des pics dans un spectre énergétique.

Pour toutes ces raisons, il me semble que l'effet qui porte son nom lui est justement attribué. Mais il est toujours passionnant d'en savoir plus sur les conditions multiples d'une découverte majeure. 